\title{
Accuracy assessment of the Non-Ideal Computational Fluid Dynamics model for siloxane MDM from the open-source SU2 suite
}

\author{
G. Gori ${ }^{\text {acc }}$, M. Zocca $^{\text {a }}$, G. Cammi ${ }^{\text {b }}$, A. Spinelli ${ }^{\text {b }}$, P.M. Congedo ${ }^{c}$, A. Guardone ${ }^{\text {a,** }}$ \\ a Department of Aerospace Science \& Technology, Politecnico di Milano, Via La Masa 34, 20156 Milano, Italy \\ ${ }^{\mathrm{b}}$ Energy Department, Politecnico di Milano, Via Lambruschini 4, 20156 Milano, Italy \\ c DeFI Team (Inria Saclay Île-de-France, Ecole Polytechnique), CMAP Lab, 1 rue d'estienne d'orves, 91120 Palaiseau, France
}

\section{A R T I C L E I N F O}

\section{Article history:}

Received 21 November 2018

Received in revised form 26 June 2019

Accepted 27 August 2019

Available online 5 September 2019

\section{Keywords:}

Non-Ideal Compressible-Fluid Dynamics

Supersonic flows

Siloxane fluid MDM

SU2

Experimental-numerical assessment

ORC applications

\begin{abstract}
A B S T R A C T
The first-ever accuracy assessment of a computational model for Non-Ideal Compressible-Fluid Dynamics (NICFD) flows is presented. The assessment relies on a comparison between numerical predictions, from the open-source suite SU2, and pressure and Mach number measurements of compressible fluid flows in the non-ideal regime. Namely, measurements regard supersonic flows of siloxane MDM (Octamethyltrisiloxane, $\mathrm{C}_{8} \mathrm{H}_{24} \mathrm{O}_{2} \mathrm{Si}_{3}$ ) vapor expanding along isentropes in the close proximity of the liquid-vapor saturation curve. The model accuracy assessment takes advantage of an Uncertainty Quantification (UQ) analysis, to compute the variability of the numerical solution with respect the uncertainties affecting the test-rig operating conditions. This allows for an uncertainty-based assessment of the accuracy of numerical predictions. The test set is representative of typical operating conditions of Organic Rankine Cycle systems and it includes compressible flows expanding through a converging-diverging nozzle in mildly-to-highly non-ideal conditions. All the considered flows are well represented by the computational model. Therefore, the reliability of the numerical implementation and the predictiveness of the NICFD model are confirmed.
\end{abstract}

(c) 2019 Elsevier Masson SAS. All rights reserved.

\section{Introduction}

Non-Ideal Compressible-Fluid Dynamics (NICFD) is the branch of fluid mechanics concerned with the investigation of fluids that do not generally abide by the Equation of State (EoS) for ideal dilute gases $P v=R T$, with $P$ pressure, $v$ specific volume, $R$ gas constant and $T$ temperature. For this reason, these fluid flows are commonly referred to as non-ideal. The non-ideal behavior is typical of molecular complex compound in the vapor phase and it becomes evident as the state of the fluid approaches the liquid-vapor saturation curve, near the critical point.

Nowadays there exists only a few Computational Fluid Dynamics (CFD) tools which are suitable for dealing with non-ideal flows. Indeed, the vast majority of numerical solvers soundly rely on the general assumption of that the fluid obeys the ideal gas law. Given that this latter assumption holds in most circumstances and applications for which a CFD solver is designed, the performances of the software can be optimized by introducing a set of simplifications that hasten the execution of the program and slim down the encoding phase. For instance, the fact that the speed of sound and the specific internal energy and enthalpy are functions of the fluid temperature only greatly simplifies CFD

\footnotetext{
* Corresponding author.

E-mail address: alberto.guardone@polimi.it (A. Guardone).
}

algorithms. Therefore, the structure of a typical CFD solver must be largely modified, or possibly even re-designed, in order to accommodate the more accurate - but unfortunately also more complex and computationally expensive - EoS required to model the non-ideal thermodynamic behavior with a reasonable level of accuracy.

The existing NICFD software represent one of the most powerful resource that researchers and engineers may use to investigate non-ideal compressible-fluid flows and to design machinery operating in the NICFD regime. Due to the demanding operating conditions, which make experimental investigations rather complicated to carry out, literature is in short supply of data regarding flows of fluids in the non-ideal regime. Only a few noteworthy papers concerning the experimental investigation of non-ideal flows were published in the past. These regard the investigation of single-phase and two-phase flows of simple compounds such as carbon dioxide $\mathrm{CO}_{2}$, see $[1,2]$. Therefore, due to the lack of experimental data in the open literature, no NICFD model in a CFD code was ever assessed against experimental data. The accuracy of the simulations, which amplifies the unknown accuracy of the underlying thermodynamic models, is therefore highly questionable.

The SU2 suite is an open-source platform designed to solve multi-physics Partial Differential Equation (PDE) problems and PDE-constrained optimization problems $[3,4]$. Recently, SU2 was 
generalized to deal with non-ideal, fully turbulent, flows and it now embodies the reference among NICFD solvers. In the limited framework of ideal flows of air, the reliability of the SU2 suite was already extensively assessed in earlier works, see for instance [3-7]. Preliminary verification of the SU2 NICFD solver implementation can be found in [8-10].

Nevertheless, none of the currently available NICFD CFD codes was ever validated against real measurements of compressible fluid flows in the NICFD regime. Recently, experimental data regarding the investigation of the influence of dense gas effects on trailing-edge loss in supersonic flows became available, see [11]. In [11], the authors use a novel Ludwieg tube facility for the study of dense-gas flows. In the very same work, the authors claim to achieve the validation of a CFD solver for non-ideal flows even if a comprehensive treatment of the numerical uncertainties (for instance the evaluation of the truncation error resulting from the mesh) is missing. In this respect, the software validation procedure reported in [11] is at least questionable.

According to [12], a fundamental step of model validation is the assessment of its accuracy. This process aims at evaluating the capability of a computational model in predicting a specified physical phenomenon. In this paper, we present the first-ever accuracy assessment of a computational model for NICFD flows against experimental data. This should not be intended as the validation of the computational solver. As mentioned earlier, the validation of the solver would require a much more comprehensive treatment of the numerical uncertainties. In other words, this papers aims at assessing the accuracy of the computational model designed to reproduce a specific experiment and it can be seen as a preliminary step towards a comprehensive validation of the non-ideal CFD solver. Hereinafter, we refer to the NICFD model as the computerized model implementing the conceptual model of NICFD flows, see [12].

The experiments concern non-ideal supersonic flows of siloxane MDM (Octamethyltrisiloxane, $\mathrm{C}_{8} \mathrm{H}_{24} \mathrm{O}_{2} \mathrm{Si}_{3}$ ) vapor in planar converging-diverging nozzles. Experimental data were collected at the Compressible-fluid dynamics for Renewable Energy Applications (CREA) laboratory of Politecnico di Milano, using the Test-Rig for Organic VApours (TROVA) $[13,14]$. The test set is representative of applications of practical interest, more precisely, of supersonic flows within the turbine vanes of Organic Rankine Cycle (ORC) power systems. Here, the model accuracy assessment is achieved by comparing experimental measurements and UQ-based predictions from the SU2 NICFD solver. The latter are obtained by including the effect of the system uncertainty through the simulation [15]. Note that the quantities targeted for the assessment are pressure and Mach number values at selected locations along the nozzle centerline.

Several papers addressed the problem of quantifying uncertainties in the numerical simulation of non-ideal flows. Some of these papers [16-18] specifically focused the attention on the uncertainties related to the thermodynamic models. In other works [19-21], multiple sources of uncertainties have been taken into account, both on operating conditions and thermodynamic models. A common finding in these works is that the uncertainties on the operating conditions yield a much higher variability of the quantities of interest w.r.t. the uncertainties associated to the thermodynamic model. In relation with an experiment, Ref. [22] was devoted to the design of a challenging scientific experiment involving the compressible flow of a dense gas in the FAST shock tube facility [23]. The expected output of the flow problem (an unconventional rarefaction shock wave) was shown to be very sensitive to uncertainties in the initial experimental conditions. This behavior drives the choices on the uncertainty characterization followed in this paper. Note anyway that all the works referred above do not include any assessment of a computational model with respect to experimental data.
This paper is structured as follows: in Section 2 the main features of the NICFD CFD solver are described, including both numerical and thermodynamics aspects. Section 3 presents the UQ framework and provides a brief description of the approach employed. The accuracy assessment of predictions from the NICFD model is confirmed through the comparison of different test cases against experimental data, reported in Section 4. Eventually, Section 5 briefly summarizes the outcome of this paper.

\section{Numerical modeling of non-ideal compressible-fluid flows}

In the following, the main features of the NICFD solver implemented in SU2 are briefly presented. Special focus is devoted to highlight differences with respect to well-known idealgas solvers. We start by recalling the three-dimensional NavierStokes equations for compressible flows, namely

$\frac{\partial \mathrm{u}}{\partial t}+\nabla \cdot \mathbf{f}(\mathrm{u})=\nabla \cdot \mathbf{d}(\mathrm{u})$

where $\mathbf{u}=\left(\rho, \boldsymbol{m}, E^{\mathrm{t}}\right)^{\mathrm{T}}$ is the vector of the conservative variables mass density $\rho \in \mathbb{R}^{+}$, momentum density $\boldsymbol{m} \in \mathbb{R}^{3}$, and total energy density per unit volume $E^{\mathrm{t}} \in \mathbb{R}, E^{\mathrm{t}}=\rho\left(e+\frac{1}{2}\|\boldsymbol{v}\|^{2}\right)$, being $e$ the internal energy per unit mass and $\boldsymbol{v}=\boldsymbol{m} / \rho$ the velocity vector. The function $\mathrm{f}(\mathrm{u}) \in \mathbb{R}^{5} \times \mathbb{R}^{3}$ is the hypervector of the advection and pressure fluxes. Namely, $\mathbf{f}(\mathrm{u})=(\boldsymbol{m}, \quad(\boldsymbol{m} \otimes$ $\left.\boldsymbol{m}) / \rho+P \boldsymbol{I}, \boldsymbol{m}\left(E^{\mathrm{t}}+P\right) / \rho\right)^{\mathrm{T}}$, where $P=P(\mathrm{u})$ is the pressure as a function of the conserved variables $u$ and $I \in \mathbb{R}^{3}$ the identity matrix. The viscous and thermal flux function $\mathbf{d}(\mathrm{u}) \in \mathbb{R}^{5} \times \mathbb{R}^{3}$ reads $\mathbf{d}(\mathrm{u})=\left(\mathbf{0}, \boldsymbol{\Pi}, \boldsymbol{v}^{\mathrm{T}} \cdot \boldsymbol{\Pi}-\boldsymbol{q}\right)^{\mathrm{T}}$. For Newtonian fluids of interest here, the viscous stress tensor $\Pi, \Pi=\Pi(v) \in \mathbb{R}^{3}$, reads $\Pi(v)=$ $\mu\left[\nabla \cdot v+(\nabla \cdot v)^{\mathrm{T}}\right]+\lambda(\nabla \cdot v) \boldsymbol{I}$, where $\mu=\mu(\mathrm{u})$ and $\lambda=\lambda(\mathrm{u})$ are the viscosity coefficients. $\boldsymbol{q}=\boldsymbol{q}(\mathrm{u})$ is the thermal flux which, under the Fourier law, simplifies to $\boldsymbol{q}(\mathrm{u})=-\kappa \nabla T(\mathrm{u})$ where $\kappa=$ $\kappa(\mathrm{u})$ is the thermal conductivity coefficient and $T=T(\mathrm{u})$ is the temperature. As widely known, the Navier-Stokes equations (1) must be closed by suitable thermodynamic models. Additionally, mathematical relations are also needed to model the molecular transport coefficients $\mu, \lambda$ and $\kappa$.

According to the State Principle, the local state of a thermodynamic system in a stable thermodynamic and chemical equilibrium is completely defined by two independent variables only. As stated in [24], a complete thermodynamic model of the fluid at equilibrium is obtained from two independent EoS, such as for example the pressure and energy EoS using the temperature $T$ and the specific volume $v=1 / \rho$ as independent variables, namely, $P=P(T, v)$ and $e=e(T, v)$. For an ideal gas $P(T, v)=$ $R T / v$ and the compatible energy EoS is a function of the temperature only. Under the further assumption of constant specific heats, the energy EoS for an ideal gas reads $e(T)=R T /(\gamma-1)$, where $\gamma>1$ is the ratio of the specific heats at constant volume and pressure, respectively. Equivalently, for fluids not obeying the ideal gas law, a complete thermodynamic description is obtained from the expression of a single thermodynamic potential, such as for example the Helmholtz energy $a(T, \rho)$. In completing the Navier-Stokes system (1) with thermodynamics, one note that the two independent variables (density and internal energy) can be immediately computed from the conservative variables vector and from the definition of total energy. Unless very simple models are considered, the computation of the thermodynamic state from $e$ and $\rho$ usually requires the solution of a non-linear equation or an equation system, since most available thermodynamic models uses the temperature and the density/specific volume as independent variables. As a consequence, the completion of the Navier-Stokes equations by means of accurate fluid models is a complex task. If compared to the ideal-gas counterpart, a non-ideal closure leads to a sharp increase of the computational time required to solve the thermodynamic problem. Within 
the SU2 framework, an embedded thermodynamic library provides access to several different EoS. Namely, it includes the Polytropic Ideal Gas (PIG) model, the polytropic van der Waals cubic EoS (VDW) [25] and the polytropic Peng-Robinson EoS (PR) [26]. Moreover, the multi-purpose thermodynamic library FluidProp [27] can be accessed through a dedicated interface which provides state-of-the-art fluid models. A set of models for the computation of molecular transport quantities in both dilute-gas and non-ideal conditions is also available within the FluidProp library. These models are based on the generalized multiparameter correlations from [28].

In turbulent flows, the transport coefficients $\mu, \lambda$ and $\kappa$ appearing in the Reynolds-Averaged Navier-Stokes (RANS) equations include also the contribution of the so-called turbulence or eddy viscosity and the total thermal conductivity resulting from the Boussinesq hypothesis, see [29]. The turbulent contribution is evaluated using well-established closures, see [4].

The modifications to generalize the SU2 solver to NICFD, which amounted to the inclusion of state-of-the-art thermodynamics model into the software, are described in [8]. Since SU2 was originally developed to deal with ideal flows only, a large portion of the code was modified or completely re-written. For example, the non-ideal dependence of the internal energy and enthalpy on both the temperature and the density - for an ideal gas both $e$ and $h$ depends on $T$ only - forbids one to use simplified expressions for the flux function $\mathbf{f}$ and its Jacobian $\mathbf{A}=\partial \mathbf{f} / \partial \mathbf{u}$. Also, transport coefficients in non-ideal conditions no longer depend on the temperature $T$ only.

A further modification is the implementation of a generalized approximate Riemann solver (ARS) of Roe type [30-32] to produce an upwind discrete counterpart of the flux function $f$ in the NICFD regime. The generalized Roe matrix $\tilde{A}_{i j}^{\eta}$ fulfilling the Roe linearization problem

$\left[\mathbf{f}\left(\mathrm{u}_{j}\right)-\mathbf{f}\left(\mathrm{u}_{i}\right)\right] \cdot \boldsymbol{\eta}=\tilde{\mathrm{A}}_{i j}^{\eta}\left[\mathrm{u}_{j}-\mathrm{u}_{i}\right]$,

between state $i$ and state $j$ along a given direction vector $\eta$, is the scalar product of the Jacobian matrix of the fluxes times the vector $\eta$. The Jacobian is evaluated at the intermediate generalized state comprising the Roe-averaged values of the velocity $\tilde{\boldsymbol{v}}_{i j}$ and total enthalpy per unit mass $\tilde{h}_{i j}^{\mathrm{t}}$. Namely,

$\tilde{\boldsymbol{v}}_{i j}=\frac{\sqrt{\rho_{i}} \boldsymbol{v}_{i}+\sqrt{\rho_{j}} \boldsymbol{v}_{j}}{\sqrt{\rho_{i}}+\sqrt{\rho_{j}}} \quad$ and $\quad \tilde{h}_{i j}^{\mathrm{t}}=\frac{\sqrt{\rho_{i}} h_{i}^{\mathrm{t}}+\sqrt{\rho_{j}} h_{j}^{\mathrm{t}}}{\sqrt{\rho_{i}}+\sqrt{\rho_{j}}}$.

In NICFD applications, an additional consistency condition must be fulfilled [32]. Namely,

$\tilde{\chi}_{i j}\left(\rho_{i}-\rho_{j}\right)+\tilde{\kappa}_{i j}\left(\rho_{i} e_{i}-\rho_{j} e_{j}\right)=\left(P_{i}-P_{j}\right)$,

where the pressure derivatives $\tilde{\chi}_{i j}$ and $\tilde{\kappa}_{i j}$ read

$\chi=\left(\frac{\partial P}{\partial \rho}\right)_{\rho e}=\left(\frac{\partial P}{\partial \rho}\right)_{e}-\frac{e}{\rho}\left(\frac{\partial P}{\partial e}\right)_{\rho}$ and

$\kappa=\left(\frac{\partial P}{\partial \rho e}\right)_{\rho}=\frac{1}{\rho}\left(\frac{\partial P}{\partial e}\right)_{\rho}$.

The approach proposed in [31] was used here to fulfill the consistency condition (3). A limited construction of the flow variables, according to the MUSCL approach, is available to increase the first-order accuracy of the numerical scheme and to obtain a second-order accuracy in smooth flow regions.

The discrete form of (1) is finally obtained by enforcing the integral form of the Navier-Stokes equations over a discrete number of node-centered finite volumes, which are constructed from the underlying unstructured triangulation of the domain. The finite volumes are baricentrical [33] and, as such, they are easily built from hybrid meshes made of elements of different types.
Furthermore, SU2 implements special boundary conditions, the so-called Riemann boundary conditions [8]. The implemented approach is able to automatically detect inflow/outflow boundaries for hyperbolic systems. The number of physical variables that can be imposed freely at a boundary depends on the way characteristic waves propagate across the domain. Namely, an inbound wave transports a given information from the outer to the inner domain whereas an outbound wave does the opposite. Only information carried by inbound waves can be arbitrarily imposed at boundaries. Boundary conditions of Riemann type can automatically detect inbound/outbound characteristics based on an eigenvalue analysis of the hyperbolic system of the Euler model. Thanks to this analysis, the correct number of enforceable unknowns is determined and the associated flow properties are therefore imposed.

The spectral decomposition of the generalized Roe matrix $\tilde{\mathrm{A}}_{i j}^{\eta}$ fulfilling the Roe linearization problem yields $\tilde{A}_{i j}^{\eta}=L \Lambda R$. Where $L$, $\mathrm{R}$ and $\Lambda$, respectively the left and right eigenvectors matrices and the diagonal matrix containing the eigenvalues $\hat{\lambda}$, are evaluated locally, at each boundary node. Note that we use the $\hat{\imath}$ notation to differentiate an eigenvalue $\hat{\lambda}$ from the viscosity coefficient $\lambda$. By analyzing the sign of the eigenvalues contained in the $\Lambda$ diagonal matrix, we can establish which are the variables we can impose at the node. Therefore, we introduce the characteristic variables jump (5),

$\delta \mathrm{w} \equiv \mathrm{R} \delta \mathrm{u}=\mathrm{R}\left(\mathrm{u}_{e}-\mathrm{u}_{i}\right)$,

where the quantity $\delta \mathrm{u}=\mathrm{u}_{e}-\mathrm{u}_{i}$ is the difference between the outer conservative vector $\mathrm{u}_{e}$ (in other word a vector containing the imposed boundary state) and the solution $u_{i}$ at the $i$ th boundary node. Negative eigenvalues are associated to inbound characteristics. Therefore, the corresponding outer state variable can be imposed. Non-negative eigenvalues are instead associated to outbound waves. This means that the related outer variable cannot be imposed and that its value at the boundary node $\mathrm{u}_{i}$ must be computed.

Therefore, we define a new operator $\psi(\hat{\lambda})$

$\psi_{k}=\psi\left(\hat{\lambda}_{k}\right)= \begin{cases}1 & \text { if } \hat{\lambda}_{k}<0 \\ 0 & \text { if } \hat{\lambda}_{k} \geq 0\end{cases}$

to obtain $\delta \mathrm{w}_{k}=\psi_{k}\left(\mathrm{R}\left[\mathrm{u}_{e}-\mathrm{u}_{i}\right]\right)$. The boundary value of the unknown, which is used to compute the numerical fluxes at the boundary, can be expressed through the following relation

$\mathrm{u}_{b}=\mathrm{u}_{i}+\mathrm{L} \operatorname{diag}\left(\psi_{k}\right) \mathrm{R}\left[\mathrm{u}_{e}-\mathrm{u}_{i}\right]$.

Since this approach is able to identify inflow/outflow boundaries in an automated manner, it turns out to be very useful when dealing with problems for which the inflow and the outflow boundaries cannot be identified a priori.

\section{Uncertainty quantification}

CFD simulations return deterministic predictions of the flow field developing within a given domain. The CFD solution is computed relying on the discretized form of a specific mathematical model and based on particular conditions imposed at boundaries or at the initial time instant. Unfortunately, our knowledge of reality is not deterministic since it is unavoidably affected by uncertainties of both aleatory and epistemic type. One of the goal of Uncertainty Quantification is to account for such uncertainties (on both parameters and measurements) in order to quantify the statistical variability of a quantity of interest.

In this paper, the uncertainties affecting the test-rig operating conditions, which are known up to measure accuracy, are propagated through the computational model of the TROVA test 
Table 1

Discharge 1. Nominal operating conditions $P^{\mathrm{t}}$ and $T^{\mathrm{t}}$ and the corresponding $2 \sigma$ confidence range.

\begin{tabular}{|c|c|c|c|c|c|c|}
\hline \multirow[t]{2}{*}{ Exp } & \multicolumn{2}{|c|}{ Total pressure } & \multicolumn{2}{|c|}{ Total temperature } & \multicolumn{2}{|c|}{ FluidProp values } \\
\hline & $P^{\mathrm{t}}[\mathrm{Pa}]$ & $U_{P}$ & $T^{\mathrm{t}}[\mathrm{K}]$ & $U_{T^{\mathrm{t}}}$ & $\mathrm{Z}[-]$ & $\gamma[-]$ \\
\hline$A_{1}$ & 919900 & 911.1 & 540.68 & 1.2 & 0.633 & 1.0178 \\
\hline$B_{1}$ & 850800 & 912.6 & 547.53 & 1.2 & 0.700 & 1.0176 \\
\hline$C_{1}$ & 624000 & 912.1 & 548.46 & 1.2 & 0.801 & 1.0176 \\
\hline$D_{1}$ & 327200 & 911.0 & 543.76 & 1.2 & 0.900 & 1.0177 \\
\hline$E_{1}$ & 83700 & 911.2 & 537.38 & 1.2 & 0.975 & 1.0178 \\
\hline
\end{tabular}

Table 2

Discharge 2. Nominal operating conditions $P^{\mathrm{t}}$ and $T^{\mathrm{t}}$ and the corresponding $2 \sigma$ confidence range.

\begin{tabular}{|c|c|c|c|c|c|c|}
\hline \multirow[t]{2}{*}{ Exp } & \multicolumn{2}{|c|}{ Total pressure } & \multicolumn{2}{|c|}{ Total temperature } & \multicolumn{2}{|c|}{ FluidProp values } \\
\hline & $P^{\mathrm{t}}[\mathrm{Pa}]$ & $U_{P \mathrm{t}}$ & $T^{\mathrm{t}}[\mathrm{K}]$ & $U_{T^{\mathrm{t}}}$ & $\mathrm{Z}[-]$ & $\gamma[-]$ \\
\hline$A_{2}$ & 458600 & 921.6 & 512.57 & 1.2 & 0.810 & 1.0183 \\
\hline$B_{2}$ & 396000 & 921.3 & 519.45 & 1.2 & 0.850 & 1.0183 \\
\hline$C_{2}$ & 268900 & 920.3 & 515.97 & 1.2 & 0.900 & 1.0182 \\
\hline$D_{2}$ & 129000 & 919.8 & 504.33 & 1.2 & 0.950 & 1.0185 \\
\hline$E_{2}$ & 52200 & 919.8 & 502.88 & 1.2 & 0.980 & 1.0185 \\
\hline
\end{tabular}

section. This is done to compute statistical moments of the selected quantities which are ultimately used to assess the accuracy of the computational model. This allows for a more robust and more comprehensive comparison of numerical and experimental results. In the following, we illustrate the characterization of the uncertainties related to the specific problem of interest here (Section 3.1). In Section 3.2 we summarize the most relevant features of the standard UQ approach we implemented to forward-propagate the uncertainties.

\subsection{Uncertainty characterization}

In this work, we exploit the whole set of data which comes from the first-ever experimental campaign aimed at investigating siloxane MDM flows expanding in the non-ideal regime [34-37]. Data were collected using the TROVA test-rig $[13,14,38]$. In the experiment, diverse quantities are measured within the settling chamber ahead of the test section and at selected locations within the test section itself. Since we are dealing with a real apparatus, the collected data surely suffer from an intrinsic uncertainty.

Namely, piezo-resistive transducers (Kulite XTEH-7L series with full scale in the range 3.5-40 bar) are employed to measure the stagnation pressure (in the settling chamber) and the static pressure at discrete locations (in the test-section). Transducers are calibrated both in pressure and temperature. A comprehensive treatment of the applied calibration procedure can be found in [34]. Namely, sensors were calibrated in the pressure range spanning from 1 bar to the full scale value and, in the temperature range, from $298 \mathrm{~K}$ to $570 \mathrm{~K}$. After calibration, an expanded Gaussian uncertainty of $0.07 \%$ of the full scale $(2 \sigma$ confidence interval) was obtained for all transducers.

Stagnation temperature is also measured within the settling chamber. Measurements are carried out using a J-type ( $\mathrm{Fe}-\mathrm{Cu} / \mathrm{Ni})$ thermocouple positioned at the symmetry axis of the settling chamber, at the same axial location of the total pressure tap. Calibration of thermocouples in the range $298-570 \mathrm{~K}$ results in expanded Gaussian uncertainties of $1.2 \mathrm{~K}$. Again, more information about the specific set-up can be found in [34].

Total temperature and total pressure measurements, as collected in the settling chamber for the different experiments, are reported in Tables 1 and 2 together with their related $2 \sigma$ confidence ranges. The latter represent the uncertainties related to the condition of the fluid entering the test-section i.e., the
Table 3

Discharge 1. Experimental pressure measurements at station p1-p4 and related expanded uncertainty ( $2 \sigma$ confidence range).

\begin{tabular}{llllll}
\hline Exp & Measure & \multicolumn{4}{l}{ Static pressure at probes } \\
\cline { 3 - 6 } & & $52.4[\mathrm{~mm}]$ & $69.4[\mathrm{~mm}]$ & $86.4[\mathrm{~mm}]$ & $103.4[\mathrm{~mm}]$ \\
\hline \multirow{2}{*}{$A_{1}$} & $P^{\mathrm{s}}[\mathrm{Pa}]$ & 876700 & 799500 & 629700 & 388800 \\
& $U_{P^{s}}[\mathrm{~Pa}]$ & \pm 1518 & \pm 1436 & \pm 317.0 & \pm 336.1 \\
\hline \multirow{2}{*}{$B_{1}$} & $P^{s}[\mathrm{~Pa}]$ & 808100 & 734500 & 573800 & 354000 \\
& $U_{P^{s}}[\mathrm{~Pa}]$ & \pm 1518 & \pm 1438 & \pm 316.9 & \pm 335.9 \\
\hline \multirow{2}{*}{$C_{1}$} & $P^{s}[\mathrm{~Pa}]$ & 589400 & 532400 & 412400 & 250800 \\
& $U_{P^{s}}[\mathrm{~Pa}]$ & \pm 1518 & \pm 1442 & \pm 317.9 & \pm 336.8 \\
\multirow{2}{*}{$D_{1}$} & $P^{\mathrm{s}}[\mathrm{Pa}]$ & 308100 & 276200 & 211000 & 126500 \\
& $U_{P^{s}}[\mathrm{~Pa}]$ & \pm 1517 & \pm 1460 & \pm 320.6 & \pm 339.9 \\
\hline \multirow{2}{*}{$E_{1}$} & $P^{\mathrm{s}}[\mathrm{Pa}]$ & 792000 & 701400 & 527100 & 311600 \\
& $U_{P s}[\mathrm{~Pa}]$ & \pm 1516 & \pm 1477 & \pm 323.5 & \pm 343.4 \\
\hline
\end{tabular}

Table 4

Discharge 2. Experimental pressure measurements at station p1-p4 and related expanded uncertainty ( $2 \sigma$ confidence range).

\begin{tabular}{llllll}
\hline Exp & Measure & \multicolumn{5}{l}{ Static pressure at $\mathrm{l}$} \\
\cline { 3 - 6 } & & $69.4[\mathrm{~mm}]$ & $86.4[\mathrm{~mm}]$ & $103.4[\mathrm{~mm}]$ & $120.4[\mathrm{~mm}]$ \\
\hline \multirow{2}{*}{$A_{2}$} & $P^{\mathrm{s}}[\mathrm{Pa}]$ & 391500 & 301600 & 182000 & 147200 \\
& $U_{P^{\mathrm{s}}}[\mathrm{Pa}]$ & \pm 1666 & \pm 349.5 & \pm 370.2 & \pm 423.7 \\
\hline \multirow{2}{*}{$B_{2}$} & $P^{\mathrm{s}}[\mathrm{Pa}]$ & 336600 & 258600 & 155200 & 125800 \\
& $U_{P \mathrm{~s}}[\mathrm{~Pa}]$ & \pm 1664 & \pm 349.6 & \pm 370.1 & \pm 423.7 \\
\hline \multirow{2}{*}{$C_{2}$} & $P^{\mathrm{s}}[\mathrm{Pa}]$ & 227500 & 173400 & 103400 & 84300 \\
& $U_{P \mathrm{~s}}[\mathrm{~Pa}]$ & \pm 1665 & \pm 349.9 & \pm 370.8 & \pm 423.7 \\
\hline \multirow{2}{*}{$D_{2}$} & $P^{\mathrm{s}}[\mathrm{Pa}]$ & 108400 & 81800 & 48600 & 38800 \\
& $U_{P^{\mathrm{s}}}[\mathrm{Pa}]$ & \pm 1673 & \pm 352.2 & \pm 373.2 & \pm 423.6 \\
\hline \multirow{2}{*}{$E_{2}$} & $P^{\mathrm{s}}[\mathrm{Pa}]$ & 43900 & 32500 & 19200 & 14500 \\
& $U_{P \mathrm{Ps}}[\mathrm{Pa}]$ & \pm 1690 & \pm 354.4 & \pm 375.2 & \pm 423.5 \\
\hline
\end{tabular}

uncertainties on the inflow boundary conditions in CFD simulations, and are thus propagated through the numerical CFD model. Tables 3 and 4 report the experimental data sets used to carry out the accuracy assessment of the computational model. Tables report the value of static pressure, and the related uncertainty, as measured at selected location within the test section.

The TROVA is also equipped with an optical Schlieren apparatus that provides images of the density gradient field $[35,37]$ within the test section. Schlieren images can be exploited to measure the Mach number $M=\|v\| / c$ of the flow, being $c$ the speed of sound. Briefly, small flaws left over the test section walls by the machining process produce perturbations that propagate as Mach waves into the stream. Following [39], a line-detection algorithm is applied to Schlieren frames to determine the slope of Mach waves $\mu$. From the well-known relation $M=1 / \sin \mu$, direct measurement of the local Mach number is carried out. This technique suffers from diverse uncertainty sources. Namely, the Schlieren image is affected by uncertainties related to the experimental setup (for instance the alignment of optical devices), uncertainties related to the image recording system (which stores images as a set of pixels of quantized intensity) or background noise due to density gradients in the air filling the volume in between the test section and the Schlieren recording screen. On top of this, the line detection algorithm is applied to the Schlieren image. The uncertainty related to wave angle measurements is assumed to be half of the angular resolution, given that this latter quantity depends on the resolution of the Schlieren image, see [40]. As a results, numerical uncertainties add up to the experimental ones yielding quite large error bars.

Concerning the thermodynamic model, hereinafter we consider the fluid model for MDM reported in [41]. This model is based on the decomposition of the reduced Helmholtz potential into an ideal and a residual part and, in this context, it 


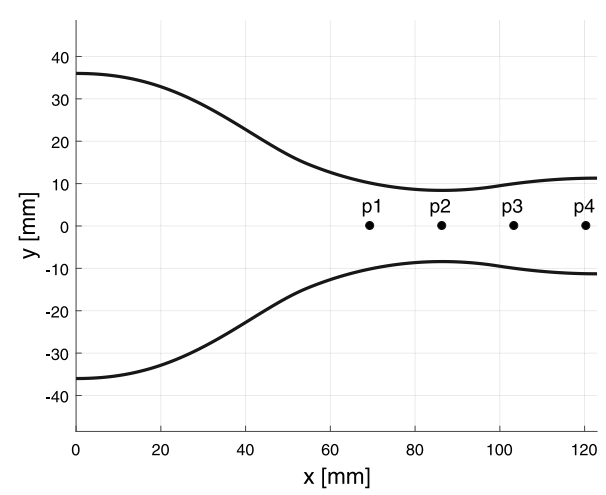

(a)

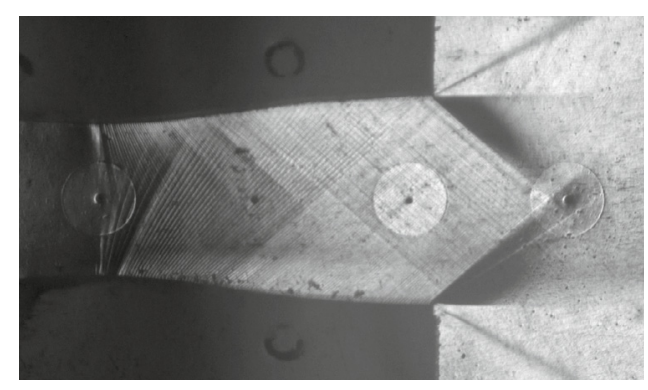

(b)

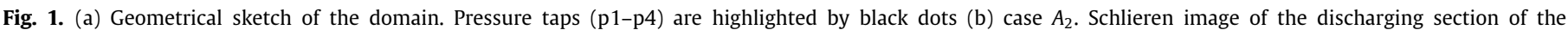
nozzle [34].

can be considered a Span-Wagner EoS optimized for MDM. As stated in [42], an EoS based on the Span and Wagner functional form can generally represent thermodynamic data within the experimental uncertainty. Moreover, the model of MDM [41] employed hereinafter is based on very accurate measurements of the $P-v-T$ saturation properties and speed-of-sound. For this reason, the employed fluid model surely represents the current state-of-the-art for siloxane MDM. In particular, we assume that the underlying uncertainties are negligible w.r.t. the ones affecting the test-rig operating conditions. Such assumption is also in accordance with several previous works [19-21] where uncertainties on the inlet operating conditions of internal NICFD flows were shown to be significantly more relevant than those related to the thermodynamic models.

\subsection{Forward uncertainty quantification method}

We illustrate here the non-intrusive strategy we employed to build polynomial chaos-based surrogate models of the computational framework. To build surrogates, and to propagate the uncertainties, we relied on the Non Intrusive Spectral Projection (NISP) library, see [43]. In this work we take advantage of a Polynomial Chaos Expansions (PCE) approach. PCE are derived from the original theory of Wiener on spectral representation of stochastic processes, using Gaussian random variables. In a nonintrusive PCE approach, a set of deterministic computations, each of which is referred to as a realization, is first carried out to obtain the exact model output for different values of the uncertain input parameters. Thanks to the solution from each realization, the coefficients of the polynomial expansion are computed and the surrogate is built.

Namely, let $\xi$ be a vector of standard independent random variables $\xi_{i}, i=1,2, \ldots, n_{\xi}$. Any well-behaved process $u$ (i.e., a second-order process with a finite variance) can be expanded in a convergent (in the mean square sense) series of the form

$$
u(\mathbf{x}, t, \xi)=\sum_{\alpha} u_{\alpha}(\mathbf{x}, t) \Psi_{\alpha}(\xi)
$$

where $\alpha$ are multi-indices, $\alpha=\left(\alpha_{1}, \alpha_{2}, \ldots, \alpha_{n}\right)$, with each component $\alpha_{i}=0,1, \ldots$, and $\Psi_{\alpha}$ being multivariate polynomial functions orthogonal with respect to the probability distribution function of the vector $\xi$. Each $\Psi_{\alpha}$ is defined by a product of orthogonal polynomials $\Phi_{i}^{\alpha_{i}}\left(\xi_{i}\right)$, that is, $\Psi_{\alpha}(\xi)=\prod_{i=1}^{n_{\xi}} \Phi_{i}^{\alpha_{i}}\left(\xi_{i}\right) . \Phi_{i}^{\alpha_{i}}$ is a polynomial of degree $\alpha_{i}$, so that the degree of $\Psi_{\alpha}$ is $|\alpha|_{1}=$ $\sum_{i=1}^{n_{\xi}} \alpha_{i}$. A one-to-one correspondence exists between the choice of stochastic variable $\xi_{i}$ and the polynomials $\Phi_{i}^{\alpha_{i}}\left(\xi_{i}\right)$. For instance, if $\xi_{i}$ is a normal/uniform variable, the corresponding $\Phi_{i}^{\alpha_{i}}\left(\xi_{i}\right)$ are

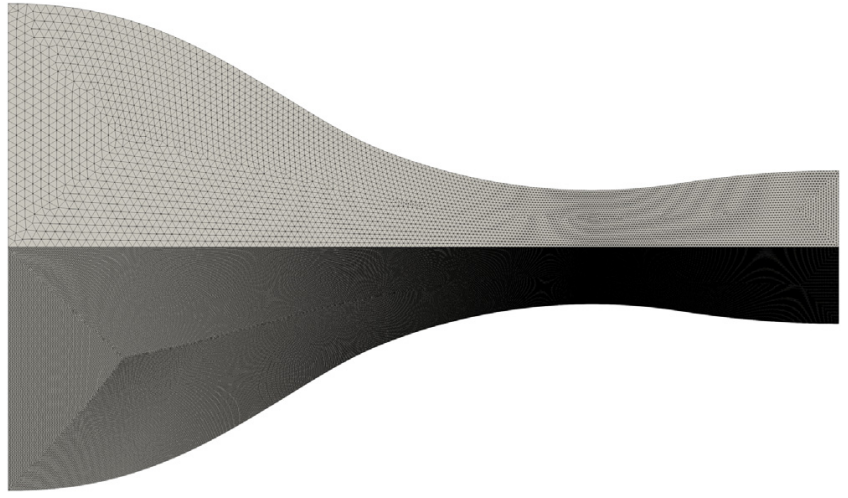

Fig. 2. Meshes of different resolution employed to carry out the grid sensitivity analysis. Upper side reports the coarsest mesh (12k elements and 6k points). Lower side reports the finest mesh (415k elements and 209k points).

Hermite/Legendre polynomials of degree $\alpha_{i}$. Coefficients $u_{\alpha}(x, t)$ are called PCE coefficients of the random process $u$. For practical use, PCEs are truncated to a degree No

$u(\mathbf{x}, t, \xi)=\sum_{|\alpha|_{1} \leq \text { No }} u_{\alpha}(\mathbf{x}, t) \Psi_{\alpha}(\xi)$

The number of multivariate polynomials $\Psi_{\alpha}$ i.e., the dimension of the expansion basis, is related to the stochastic dimension $n_{\xi}$ and the degree No of polynomials and it is given by the formula $\left(n_{\xi}+\mathrm{No}\right) ! /\left(n_{\xi} ! \mathrm{No} !\right)$.

Several approaches can be used to estimate PCE coefficients. The approach used in this study is based on quadrature formulae [43]. The PCE coefficients $u_{\alpha}(x, t)$ are evaluated from a set of abscissas and weights $\left(\boldsymbol{\xi}_{i}, \omega_{i}\right)$

$u_{\alpha}(\mathbf{x}, t)=\left\|\Psi_{\alpha}\right\|^{-2} \sum_{i=1}^{n} u\left(\mathbf{x}, t, \xi_{i}\right) \Psi_{\alpha}\left(\xi_{i}\right) \omega_{i}$,

where $n$ refers to the number of points (which depends on the quadrature formula).

From the PCE of the random process, it is then straightforward to compute the mean $(E)$ and variance $(V)$ of the random process $u(\mathbf{x}, t)$ and to ultimately estimate sensitivity information using the ANalysis Of VAriance decomposition (ANOVA) [43]

$E(u(\mathbf{x}, t))=u_{0}(\mathbf{x}, t), \quad V(u(\mathbf{x}, t))=\sum_{\alpha} u_{\alpha}^{2}(\mathbf{x}, t)$. 


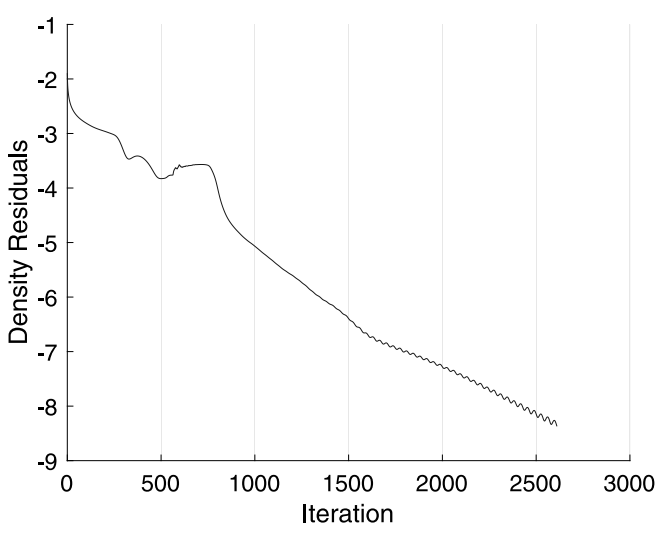

(a)

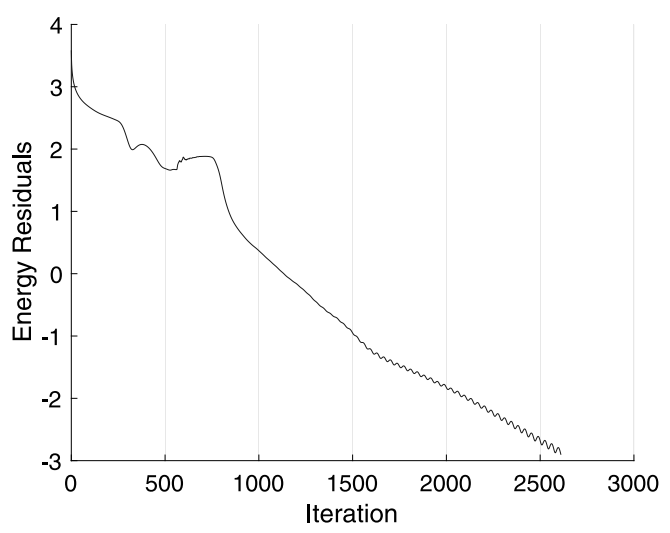

(b)

Fig. 3. Case $A_{2}$, simulation convergence histories. (a) Density residuals; (b) Energy residuals.

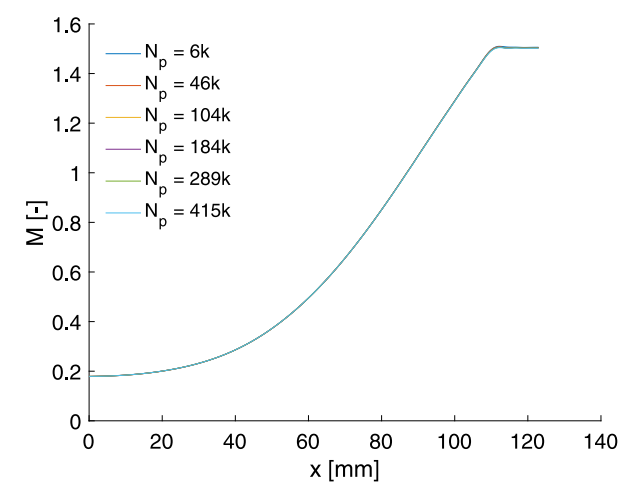

(a) Mach number

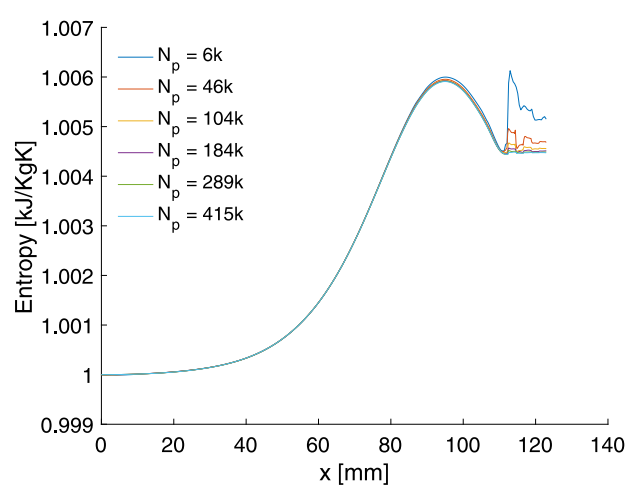

(c) Entropy

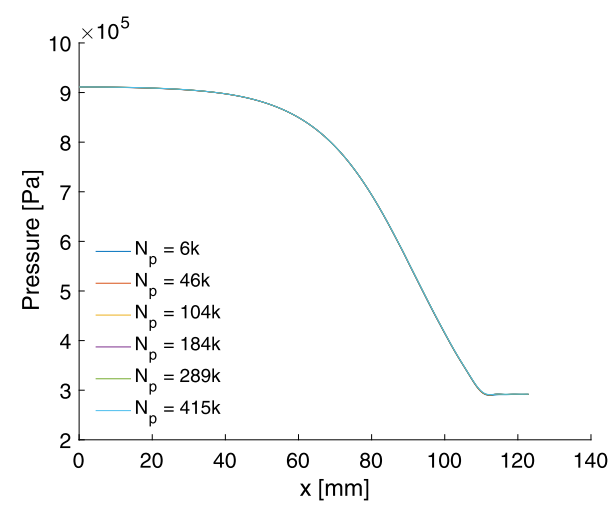

(b) Static pressure

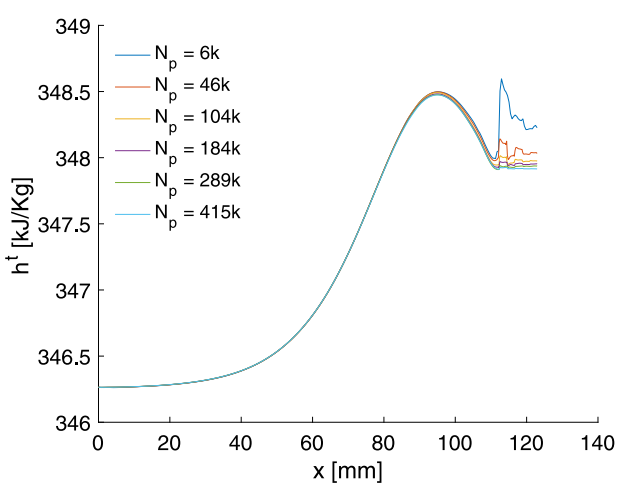

(d) Total Enthalpy

Fig. 4. Grid sensitivity analysis considering a symmetric domain. Trends are extracted along the nozzle centerline.

ANOVA relies on the decomposition of the variance according to the contribution of each uncertainty. This allows the quantification of the contribution of a given stochastic parameter to the total variance of an output quantity. Within a PCE framework, this can be easily achieved by using some interesting properties of the previous development. Indeed, the variance decomposition of the response can be written as follows:

$V(u)=\sum_{u \subset U} \sigma_{u}^{2}\left(X_{u}\right)$

where $U=\left(1,2, \ldots, n_{\xi}\right)$ is the set of random variables indexes and $\sigma_{u}^{2}$ is the variance introduced by interactions of random variables $X_{u} \subseteq X_{U}$. From a PCE, it is easy to compute all variances terms $\sigma_{u}^{2}$ of $X_{u}$ equivalent to $\xi_{u}$ i.e., $\sigma_{u}^{2}=\sum_{\alpha \geq u} u_{\alpha}^{2}$, with the notation $\alpha \geq u \Rightarrow \alpha_{i} \geq u_{i} \forall i=1,2, \ldots, n_{\xi}$. The associated sensitivity measure of $X_{u}$, named Sobol's index, is written as the correlation ratio

$S_{u}=\frac{\sum_{v \leq u} \sigma_{v}^{2}}{V(u)}$.

For more details, the reader is referred to Ref. [43]. 


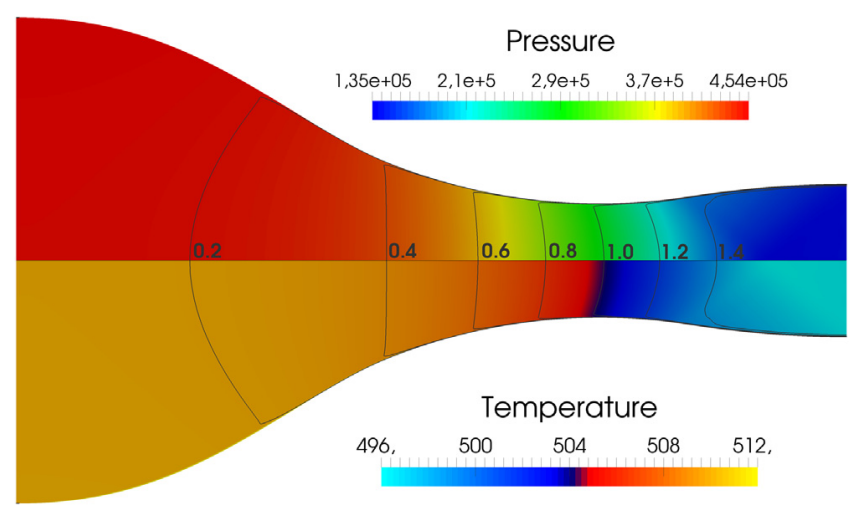

Fig. 5. Case $A_{2}$. Pressure (upper) and temperature (lower) fields as computed by the NICFD solver. Mach contours are also reported. Quantities vary smoothly along the axis of the nozzle whereas they are almost uniform over each sections.

\section{Results}

In this section we present the accuracy assessment of the CFD model developed to simulate a specific experiment carried out using the TROVA facility. Namely, the assessment is achieved by comparing predictions and measurements. The comparison is carried out exploiting an uncertainty quantification framework which is used to forward propagate the experimental uncertainties through the CFD model of the test section, see Section 3.

The TROVA is a blow-down test-rig facility specifically designed to investigate the expansion of molecular complex fluids in the NICFD region of interest for ORC applications. The fluid, siloxane MDM, is first brought in its vapor phase at conditions of high pressure and density, close to the liquid-vapor equilibrium curve. When the desired thermodynamic conditions are met, a valve is open and the fluid is conveyed into the test-section, where it accelerates up to a supersonic speed across a planar converging-diverging nozzle. As the fluid flows across the nozzle, it undergoes an almost isentropic and adiabatic expansion. Eventually, the fluid is discharged into a low pressure reservoir, where it is recovered. Further details regarding the test-rig set-up can be found in [37] and [35].

As the high-pressure reservoir empties, total conditions change and the flow within the nozzle encompasses highly nonideal to ideal conditions. In the following, we selected 5 time instants (A-E) during the TROVA discharge and we simulated the corresponding flows. At each instant, steady upstream conditions, specifically set to match those measured in the settling chamber, apply. This latter assumption is confirmed by experimental observations which confirm negligible flow unsteadiness over the considered time scale, see [37]. In [34,37,44], it is shown that the time-scale characterizing the emptying process of the high pressure reservoir is larger than the flow residence time within the nozzle by at least two orders of magnitude. Moreover, the flow thermodynamic relaxation time, see [45], is by far lower than the time-scale associated to the motion of a molecule that crosses the test-section, from the inlet to the nozzle outflow boundary. These arguments justify the steady flow assumption which, therefore, has negligible implications in the comparison of predictions with measurements. Two different discharges (discharge 1 and discharge 2) were considered, for a total number of 10 tests. Tables 1 and 2 report the total pressure and temperature as measured in the settling chamber at each time instant (A-E), respectively for discharge 1 and 2 .

To simulate the experiment, we rely on a two-dimensional representation of the test-rig, see Fig. 1(a). The same numerical set-up, described below, applies for all the simulations presented in this work. The domain is discretized using an in-house meshing tool which implements an advancing-front/Delaunay algorithm to generate unstructured grids of triangular elements. In the following, $N_{p}$ and $N_{e}$ respectively indicate the number of points and the number of elements of the primary grid. Hereinafter, numerical predictions are obtained assuming an inviscid and adiabatic flow (i.e., an Euler flow). As documented in $[34,46]$, the presence of a thin viscous layer developing on nozzle walls has negligible effects on the properties of the flow at the inner inviscid core, along the centerline. The Helmholtz EoS [41] implemented in the FluidProp library, which is currently considered the state-of-the-art for siloxane MDM, is employed in all computations. Only steady flow simulations were carried out. At the domain inflow, boundary conditions impose the value of flow total temperature and total pressure. Tables 1 and 2 summarize the inflow conditions for all the considered test cases. At the outflow, the boundary condition applies a uniform value of static pressure. Note that SU2 implements the so-called Riemann boundary conditions, see Section 2. Therefore, the code recognizes the subsonic/supersonic nature of a boundary in an autonomous manner and it is able to apply the correct condition. In all cases, the CFD solution is marched forward in time, using an implicit Euler scheme, until a steady state is reached. A second-order accurate MUSCL scheme of Roe type is employed with a flux limiting function (Venkatakrishnan flux limiter). The Green-Gauss formula is used to reconstruct gradients at cell interfaces.

For each test-case, $5^{2}$ realizations, corresponding to the locations of the tensorization of a 1D Hermite polynomials with order five in each direction, are carried out. The PCE approximation has been chosen relying on a convergence study (not reported here for brevity).

In the test section, probes for the measure of static pressure are flush mounted at selected stations along the nozzle centerline. Tables 3 and 4 report the values of the static pressure, as measured at $\mathrm{p} 1-\mathrm{p} 4$ stations along the nozzle centerline (the positions varies from discharge 1 to 2 ), together with their expanded uncertainty.

A sensitivity analysis is first carried out to evaluate the dependency of the solution w.r.t. the computational grid. Different levels of mesh resolution were chosen and the variability of a few quantities was assessed w.r.t. the spatial discretization. Note that the geometry is symmetric along the nozzle centerline. Therefore, simulations were carried out considering half domain only. For this investigation, the inflow conditions correspond to those labeled $A_{1}$. Grids are made up by an increasing number of elements: the coarser mesh counts almost $12 \mathrm{k}$ elements ( $6 \mathrm{k}$ points) while the finest one contains $415 \mathrm{k}$ elements (209k points). Fig. 2 reports a comparison of the coarsest (upper side) versus the finest (lower side) grid.

Fig. 3(a-b) show the history of density and energy residual over solver iterations, similar convergence history is found for all the grids considered in the sensitivity analysis.

Results from the sensitivity analysis are reported in Fig. 4. The Mach number and pressure distributions along the nozzle centerline, respectively Fig. 4(a-b), show a very loose dependency on the selected spatial discretization. Indeed, curves resulting from different meshes are superimposed. On the other hand, the entropy, Fig. 4(c), and the total enthalpy, Fig. 4(d), trends suffer from a certain dependency on the numerical grid. In particular, we observe two phenomena: the enthalpy and the entropy are not conserved along the nozzle centerline and there is a sudden increase, for both quantities, in the final portion of the nozzle, close to the discharge section.

Rigorously, because of the inviscid and adiabatic flow assumptions, the total enthalpy is a quantity conserved across the 


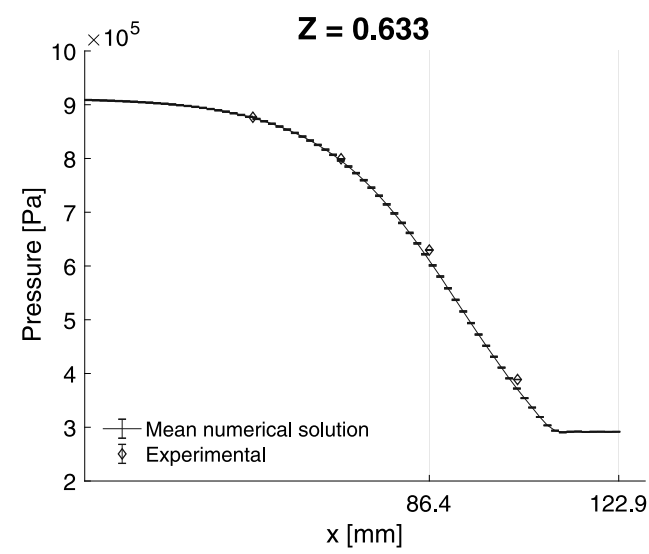

(a) $A_{1}$

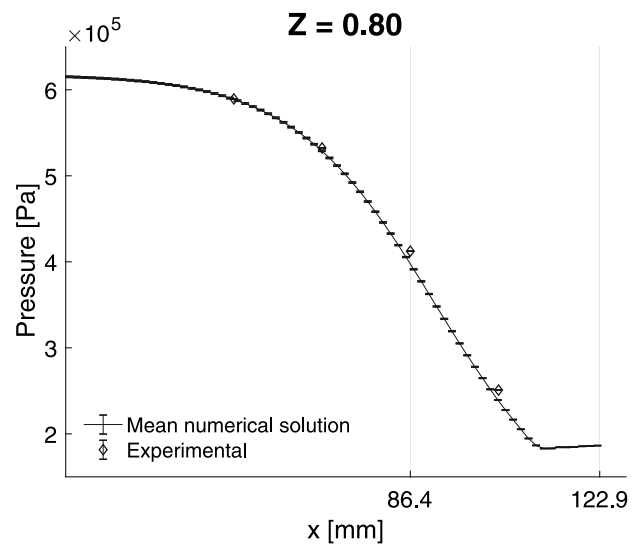

(c) $C_{1}$

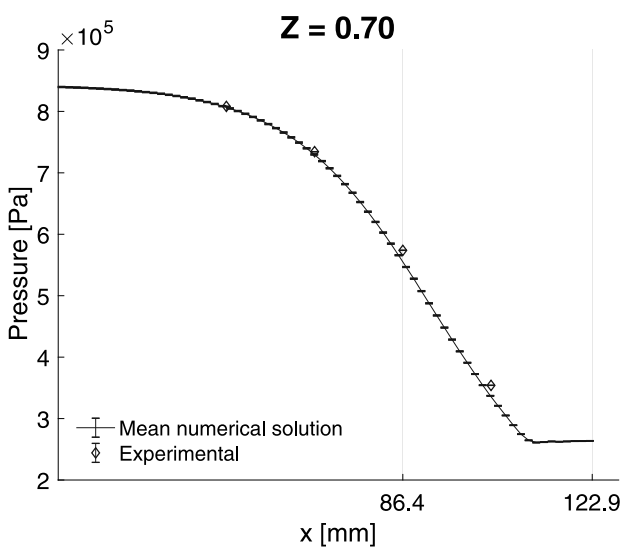

(b) $B_{1}$

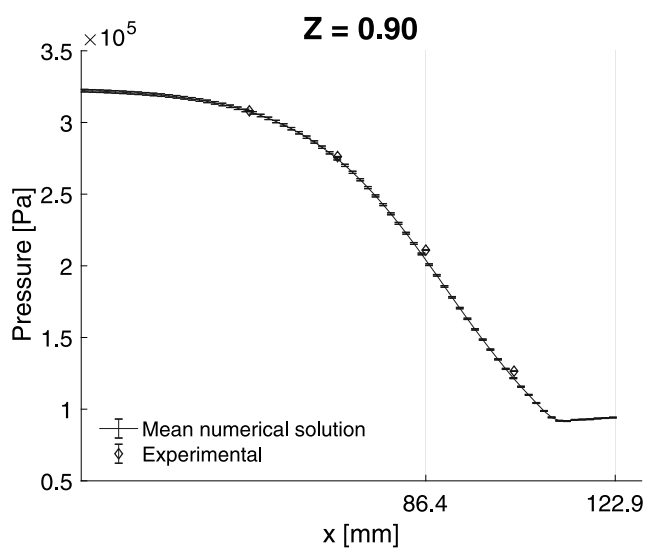

(d) $D_{1}$

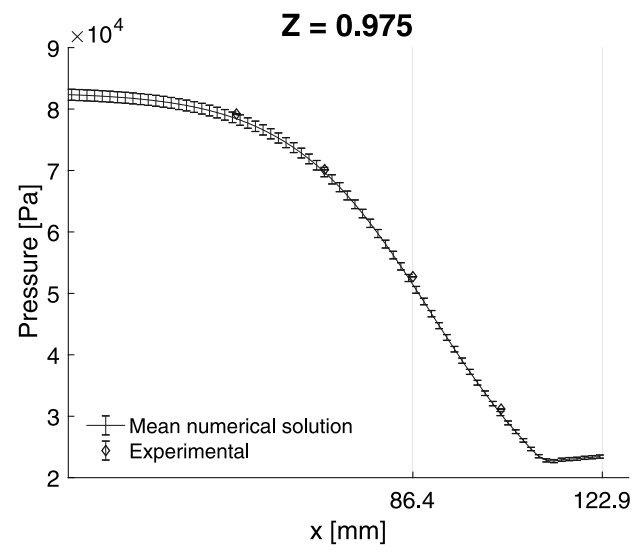

(e) $E_{1}$

Fig. 6. Discharge 1. Comparison of numerical results against experimental measurements (diamond marks).

domain. Moreover, since the test case does not include shocks or regions of separated flow, the solution is also homoentropic. In CFD simulations involving inviscid and adiabatic flows, regions of entropy increase represent important markers which provide a picture of the influence of numerical dissipation over the computed solution, see [47]. In particular, the non-constant entropy and enthalpy trends seem to be related to the strong curvature characterizing the nozzle profile. Indeed, the SU2 solver implements standard non-penetrating wall conditions and it does not provide for any special treatment of solid boundaries with a strong curvature. Curved walls are usually associated to numerical entropy generation, total pressure loss, drag, and grid convergence issue, see $[48,49]$. Nevertheless, in the considered test case results reveal a maximum variation, from the lowest to the largest value, in the order of $\approx 0.6 \%$, for both entropy and enthalpy. The error is very limited meaning that numerical dissipation effects are limited as well.

Remarkably, in the close proximity of the nozzle discharge section the entropy and enthalpy curves show a discontinuity which vanishes as the grid is refined. This seems to be related to a poor discrete representation of the nozzle profile in coarser meshes. In particular, the region where the wall curvature changes sign, namely passing from the convex throat profile to the concave divergent, appears to produce perturbations that propagate downstream into the supersonic flow. These perturbations reach the nozzle centerline at a specific point where they cause a sudden 


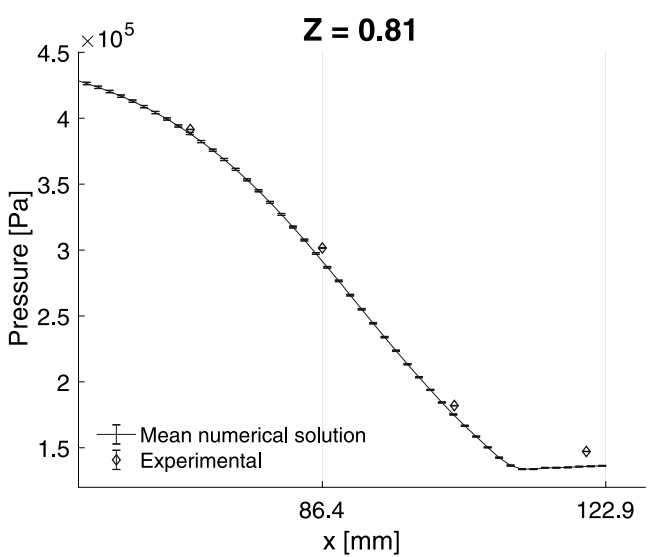

(a) $A_{2}$

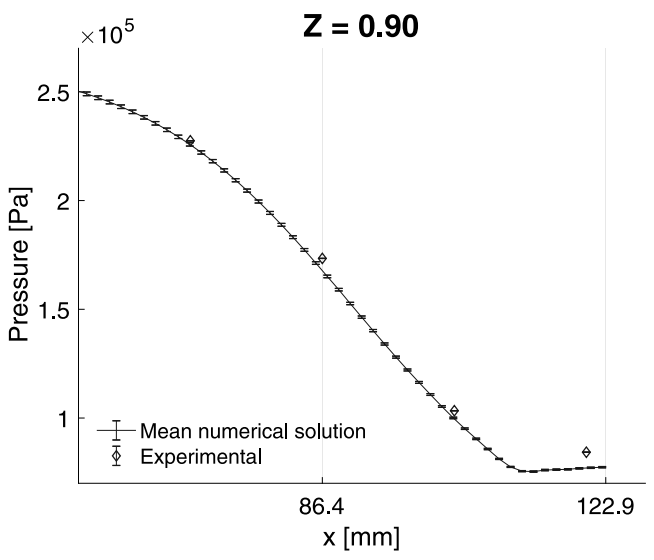

(c) $C_{2}$

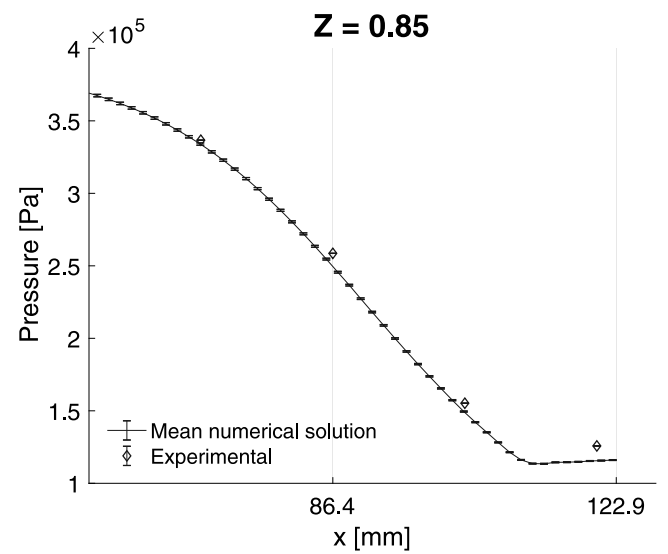

(b) $B_{2}$

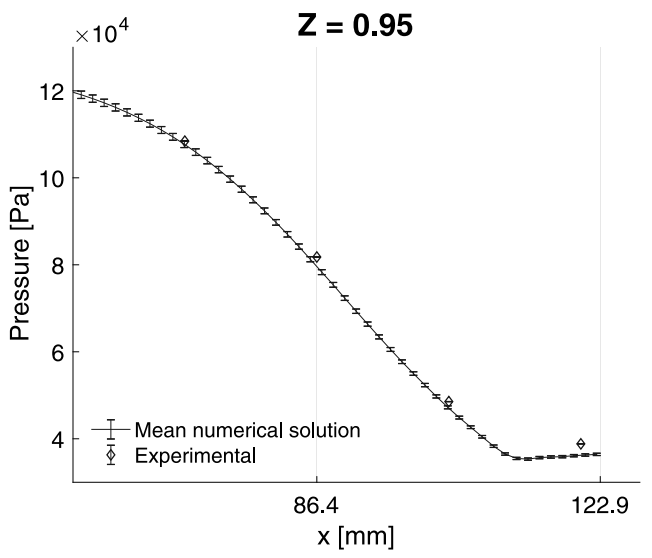

(d) $D_{2}$

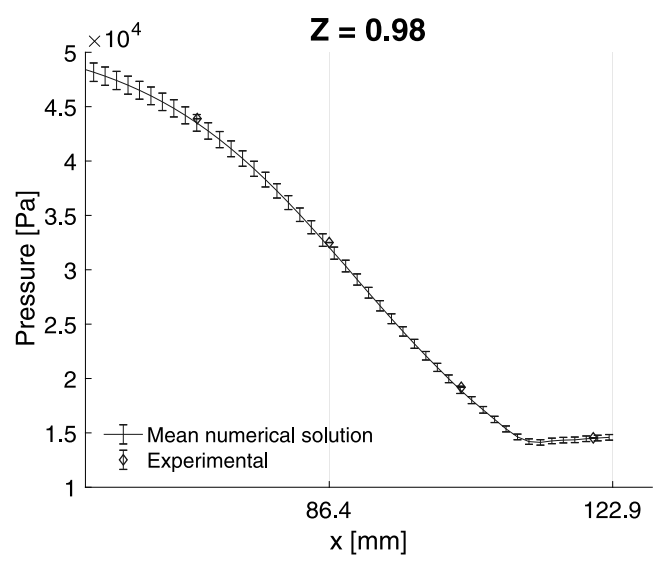

(e) $E_{2}$

Fig. 7. Discharge 2. Comparison of numerical results against experimental measurements (diamond marks).

entropy and enthalpy increase. These jumps correctly decrease as the grid resolution increases, thus the validity of numerical predictions is not questionable if a sufficiently refined grid is employed. Despite this, the sensitivity analysis guarantees that a grid independent solution is largely achieved for the targeted quantities of interest (Mach number and Pressure).

Fig. 5 reports the pressure (upper) and temperature (lower) fields computed for conditions $A_{2}$. Mach contours are also reported. The MDM flows from left to right, accelerating up to supersonic speed upon expansion. The numerical solution shows a quasi one-dimensional behavior and isolines of assume the parabolic shape expected in these kind of flows. Flow properties are found to vary smoothly along the nozzle axis.

The computational model accuracy assessment is carried out considering 10 test cases $\left(A_{1}-E_{1}\right.$ and $\left.A_{2}-E_{2}\right)$. The compressibility factor $Z=P v /(R T)$ at the inlet of the nozzle spans from 0.633 (for the most non-ideal case) to approximately 1 . Note that the compressibility factor $Z$ provides an estimation of the relevance of non-ideal effects. Indeed, for ideal gases $P v=R T$ and hence $Z \equiv 1$.

Based on the grid sensitivity analysis, we select a mesh counting $104 \mathrm{k}$ elements (53k points). The total conditions applied at the inlet are reported in Tables 1 and 2, together with the $2 \sigma$ 


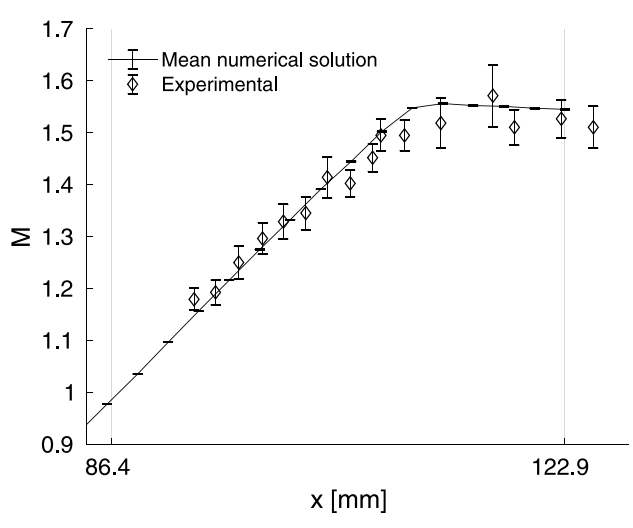

(a)

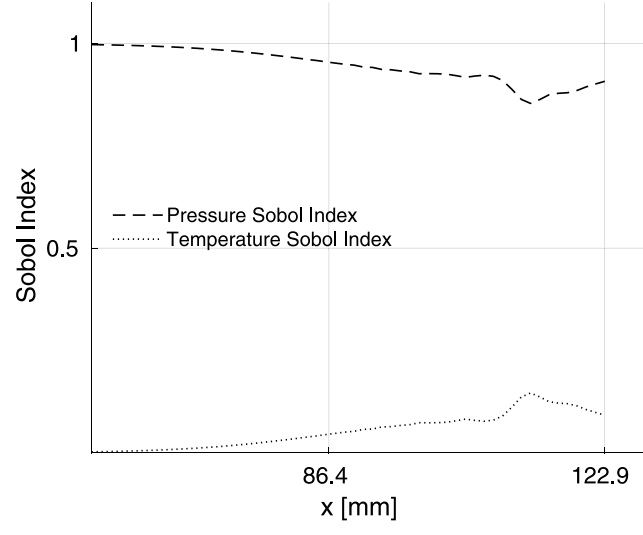

(b)

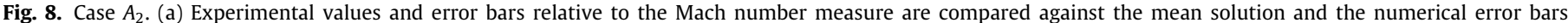
resulting from the UQ analysis. (b) Sobol indices, w.r.t. the inflow total pressure and temperature, related to static pressure predictions variance.

confidence range for each variable. Surrogate models are built on a 4-th order polynomial equation that brought to the definition of a $5 \times 5$ test matrix. Figs. $6(a-e)$ and $7(a-e)$ show the pressure mean trends obtained by propagating the inflow uncertainties using the surrogate models. For all the considered experiments, pressure trends are complemented by the numerical uncertainty bars $( \pm 2 \sigma)$ resulting from the UQ analysis. On the same plots, the experimental measurements and the corresponding uncertainties are also reported for comparison.

The numerical solution fairly matches experimental data for all the considered operating conditions. Discrepancies among numerical and experimental trends, in particular at the exhaust section, are generally found to increase as the inflow regime becomes more and more non-ideal.

Fig. 1(b) reports the Schlieren image centered on the diverging section of the nozzle (relative to the experiment run $A_{2}$ ). The Schlieren image confirms that the flow in the divergent is supersonic and that no shock waves are present in the divergent section. Indeed, two symmetrical rarefaction fans are clearly visible at the discharge section (dark triangular regions on the right hand side of Fig. 1(b)). The knife is rotated so that density gradients parallel to the nozzle axis are visualized, see [50]. Darker and lighter gray levels are associated, respectively, to positive (compressions) and negative (expansions) density gradients. In the reported frame Fig. 1(b), the portion of the domain corresponding to the nozzle throat and to the symmetric expansion fans generated at the discharge section appear as dark regions instead as bright. In [51], the authors analyzed the optical path in the TROVA Schlieren set-up by means of a ray tracing algorithm. Simulations revealed that light rays crossing regions of strong density gradients are deviated outside the measuring range. Therefore, light rays that are significantly diverted from the unperturbed path do not reach the camera sensor and expansions appear as dark regions in the Schlieren frame. As mentioned, Schlieren images of a supersonic flow can be exploited to directly measure the local value of the Mach number. Fig. 8(a) reports the experimental measurements and the related error bars, see [52]. In the same plot, the numerical mean solution and the $2 \sigma$ confidence range, resulting from the UQ analysis, are reported for comparison. The mean solution is well included within the experimental error bars, pointing out the reliability of the predicted Mach number trend. Moreover, the $2 \sigma$ range is very limited, thus confirming the robustness of the numerical solution with respect to the considered uncertainties. In Fig. 8(b), the Sobol indices of static pressure are reported. Fig. 8(b) reveals that the uncertainty related to the value of total pressure at the inlet has a major influence on the variability of the static pressure. On the other hand, Sobol coefficients show that the uncertainty on temperature at the inlet produces relevant effects in the close proximity of the discharging section only.

Both quantities, pressure and temperature, are associated to a very small variability pointing out a very robust and predictive numerical solution, with respect to the experiments.

\section{Conclusions}

For the first time, the capabilities of a Non-Ideal Computational Fluid Dynamics (NICFD) model were assessed against experimental results regarding compressible flows of siloxane MDM vapor in the non-ideal regime. Experimental data used for comparison were collected using the TROVA facility at Politecnico di Milano.

The accuracy of the computational model designed to reproduce the specific experiment was assessed for a set of exemplary NICFD flow-field that are of relevant interest to practical applications. The set includes a series of supersonic expanding flows in a planar converging-diverging nozzle representative of ORC supersonic stators.

Numerical simulations based on the Euler flow model were carried out using the non-ideal CFD solver embedded in the SU2 open-source suite. A non-intrusive polynomial chaos-based technique was used to forward propagate the physical uncertainties associated to the inlet conditions through the computational model.

Comparison against experimental data revealed that all the considered NICFD flows are fairly well predicted by the computational model. The resulting uncertainty bars were limited to very small values, pointing out the robustness and the predictive character of the numerical tool, at least concerning non-ideal flows of siloxane MDM.

Though the assessment is mostly focused on evaluating the accuracy of the computational model in a limited region of the thermodynamic state space, at conditions close to the saturation line, the considered test cases are representative of a broad range of ORCs typical operating conditions. The accuracy assessment presented in this work is therefore fundamental to ORC applications, as it gives credibility to the numerical evaluation of ORC performances.

\section{Acknowledgments}

The research is funded by the European Research Council under Grant ERC Consolidator 2013, project NSHOCK 617603. 
The authors would like to thank Prof. Fabio Cozzi, Simone Gallarini and Camilla Conti for their contribution in clarifying the experimental set-up.

\section{References}

[1] K.M. Duff, Non-Equilibrium Condensation of Carbon Dioxide in Supersonic Nozzles (Ph.D. thesis), Massachusetts Institute of Technology. Dept. of Mechanical Engineering, 1966, pp. 48-51, Thesis. 1966. Sc.D.; Bibliography: leaves.

[2] K. Bier, F. Ehrler, G. Theis, Spontaneous Condensation in Stationary Nozzle Flow of Carbon Dioxide in a Wide Range of Density, 1990, pp. 129-141.

[3] F. Palacios, M.R. Colonno, A.C. Aranake, A. Campos, S.R. Copeland, T.D. Economon, A.K. Lonkar, T.W. Lukaczyk, T.W.R. Taylor, J.J. Alonso, Stanford University Unstructured $\left(\mathrm{SU}^{2}\right)$ : An open-source integrated computational environment for multi-physics simulation and design, AIAA Paper 2013-0287 51st AIAA Aerospace Sciences Meeting and Exhibit.

[4] T.D. Economon, D. Mudigere, G. Bansal, A. Heinecke, F. Palacios, J. Park, M. Smelyanskiy, J.J. Alonso, P. Dubey, Performance optimizations for scalable implicit RANS calculations with SU2, Comput. \& Fluids 129 (2016) $146-158$.

[5] F. Palacios, T.D. Economon, A. Aranake, R.S. Copeland, A. Lonkar, T. Lukaczyk, D.E. Manosalvas, R.K. Naik, S. Padron, B. Tracey, A. Variyar, J.J. Alonso, Stanford university unstructured (SU2): Analysis and design technology for turbulent flows, AIAA Paper 2014-0243 52nd Aerospace Sciences Meeting.

[6] T.D. Economon, F. Palacios, S.R. Copeland, T.W. Lukaczyk, J.J. Alonso, SU2: An open-source suite for multiphysics simulation and design, AIAA J. 54 (3) (2015) 828-846.

[7] R. Sanchez, H. Kline, D. Thomas, A. Variyar, M. Righi, D.T. Economon, J.J. Alonso, R. Palacios, G. Dimitriadis, V. Terrapon, Assessment of the fluid-structure interaction capabilities for aeronautical applications of the open-source solver SU2, ECCOMAS, VII European Congress on Computational Methods in Applied Sciences and Engineering, Crete Island, Greece.

[8] S. Vitale, G. Gori, M. Pini, A. Guardone, T.D. Economon, F. Palacios, J.J Alonso, P. Colonna, Extension of the SU2 open source CFD code to the simulation of turbulent flows of fluids modelled with complex thermophysical laws, in: 22nd AIAA Computational Fluid Dynamics Conference, AIAA Paper 2760, 2015.

[9] G. Gori, A. Guardone, S. Vitale, A. Head, M. Pini, P. Colonna, Non-ideal compressible-fluid dynamics simulation with SU2: Numerical assessment of nozzle and blade flows for organic rankine cycle applications, in: 3rd International Seminar on ORC Power Systems, Brussels, Belgium, 2015.

[10] M. Pini, S. Vitale, P. Colonna, G. Gori, A. Guardone, T. Economon, J. Alonso, F. Palacios, SU2: The Open-Source Software for Non-Ideal Compressible Flows, Vol. 821, 2017, 012013.

[11] F.J.D. Galiana, A.P.S. Wheeler, J. Ong, C.A. de M. Ventura, The effect of dense gas dynamics on loss in ORC transonic turbines, J. Phys. Conf. Ser. 821 (1) (2017) 012021.

[12] W.L. Oberkampf, C.J. Roy, Verification and Validation in Scientific Computing, Cambridge University Press, 2010, http://dx.doi.org/10.1017/ CB09780511760396.

[13] A. Spinelli, M. Pini, V. Dossena, P. Gaetani, F. Casella, Design, simulation, and construction of a test rig for organic vapours, ASME J. Eng. Gas Turb. Power 135 (2013) 042303.

[14] A. Guardone, A. Spinelli, V. Dossena, Influence of molecular complexity on nozzle design for an organic vapor wind tunnel, ASME J. Eng. Gas Turb. Power 135 (2013) 042307

[15] C.J. Roy, W.L. Oberkampf, A comprehensive framework for verification, validation, and uncertainty quantification in scientific computing, Comput. Methods Appl. Mech. Engrg. 200 (25) (2011) 2131-2144.

[16] P. Cinnella, P. Congedo, L. Parussini, Quantification of thermodynamic uncertainties in real gas flows, Int. J. Eng. Syst. Model. Simul. 2 (1-2) (2010) 12-24.

[17] P. Cinnella, P. Congedo, V. Pediroda, L. Parussini, Sensitivity analysis of dense gas flow simulations to thermodynamic uncertainties, Phys. Fluids (23).

[18] X. Merle, P. Cinnella, Bayesian quantification of thermodynamic uncertainties in dense gas flows, Reliab. Eng. Syst. Saf. 134 (Supplement C) (2015) 305-323.

[19] P. Congedo, C. Corre, J.-M. Martinez, Shape optimization of an airfoil in a BZT flow with multiple-source uncertainties, Comput. Methods Appl. Mech. Engrg. 200 (1-4) (2011) 216-232.

[20] P. Congedo, G. Geraci, R. Abgrall, V. Pediroda, L. Parussini, TSI metamodelsbased multi-objective robust optimization, Eng. Comput. (Swansea, Wales) 30 (8) (2013) 1032-1053.
[21] G. Geraci, P. Congedo, R. Abgrall, G. Iaccarino, High-order statistics in global sensitivity analysis: Decomposition and model reduction, Comput. Methods Appl. Mech. Engrg. 301 (2016) 80-115.

[22] P. Congedo, P. Colonna, C. Corre, J. Witteveen, G. Iaccarino, Backward uncertainty propagation method in flow problems: Application to the prediction of rarefaction shock waves, Comput. Methods Appl. Mech. Engrg. 213 (2012) 314-326.

[23] P. Colonna, A. Guardone, N.R. Nannan, C. Zamfirescu, Design of the dense gas flexible asymmetric shock tube, ASME J. Fluids Eng. 130.

[24] H.B. Callen, Thermodynamics and an Introduction to Thermostatistics, second ed., Wiley, 1985.

[25] J.D. van der Waals, Over de Continul̈teit van den Gas - en Vloeistoftoestand (on the Continuity of the Gas and Liquid State) (Ph.D. thesis), Leiden University, 1873.

[26] D.Y. Peng, D.B. Robinson, A new two-constant equation of state, Ind. Eng. Chem. Fundam. 15 (1976) 59-64

[27] P. Colonna, T.P. der Stelt, A. Guardone, FluidPRop: A Program for the Estimation of Thermophysical Properties of Fluids, Energy Technology Section, Delft University of Technology, The Netherlands, 2005.

[28] T.-H.H. Chung, M. Ajlan, L.L. Lee, K.E. Starling, Generalized multiparameter correlation for nonpolar and polar fluid transport properties, Ind. Eng. Chem. Res. 27 (4) (1988) 671-679.

[29] D. Wilcox, Turbulence Modeling for CFD, second ed., DCW Industries, Inc., 1998.

[30] P. Roe, Approximate Riemann solvers, parameter vectors and difference schemes, J. Comput. Phys. 43 (1981) 357-372.

[31] J. Montagne, M. Vinokur, Generalized flux-vector splitting and Roe average for an equilibrium real gas, J. Comput. Phys. 89 (2) (1990) 276-300.

[32] A. Guardone, L. Vigevano, Roe linearization for the van der Waals gas, J. Comput. Phys. 175 (1) (2002) 50-78.

[33] V. Selmin, The node-centred finite volume approach: bridge between finite differences and finite elements, Comput. Methods Appl. Mech. Engrg. 102 (1993) 107-138.

[34] M. Zocca, Experimental Observation of Supersonic Non-Ideal CompressibleFluid Flows (Ph.D. thesis), Politecnico di Milano, 2018.

[35] A. Spinelli, A. Guardone, F. Cozzi, M. Carmine, R. Cheli, M. Zocca, P. Gaetani, V. Dossena, Experimental observation of non-ideal nozzle flow of siloxane vapor MDM, in: 3rd International Seminar on ORC Power Systems, Brussels, Belgium, 2015

[36] A. Spinelli, F. Cozzi, G. Cammi, M. Zocca, P. Gaetani, V. Dossena, A. Guardone, Preliminary characterization of an expanding flow of siloxane vapor MDM, in: 1st International Seminar on Non-Ideal Compressible-Fluid Dynamics for Propulsion and Power, Varenna, Italy, 2016.

[37] A. Spinelli, F. Cozzi, M. Zocca, P. Gaetani, V. Dossena, A. Guardone, Experimental investigation of a non-ideal expansion flow of siloxane vapor MDM, in: Proceedings of the ASME 2016 Turbo Expo, Soul, GT2016-57357, 2016.

[38] M. Pini, A. Spinelli, V. Dossena, P. Gaetani, F. Casella, Dynamic simulation of a test rig for organic vapours, in: Proceedings of $5^{\text {th }}$ Conference on Energy Sustainability, ASME EsFuelCell2011, Washington, Washington DC, USA, 2011.

[39] R. Lo, W. Tsai, Gray-scale hough trasform for thick line detection in grey-scale images, Pattern Recognit. 28 (1995) 647-661.

[40] A. Spinelli, G. Cammi, S. Gallarini, M. Zocca, F. Cozzi, P. Gaetani, V. Dossena, A. Guardone, Experimental evidence of non-ideal compressible effects in expanding flow of a high molecular complexity vapour, Exp. Fluids 59 (8) (2018) 126.

[41] M. Thol, F.H. Dubberke, E. Baumhö, J. Vrabec, R. Span, Speed of sound measurements and fundamental equations of state for octamethyltrisiloxane and decamethyltetrasiloxane, J. Chem. Eng. Data 62 (9) (2017) 2633-2648.

[42] P. Colonna, N.R. Nannan, A. Guardone, E.W. Lemmon, Multiparameter equations of state for selected siloxanes, Fluid Phase Equilib. 244 (2) (2006) $193-211$.

[43] T. Crestaux, O. Le Matre, J.-M. Martinez, Polynomial chaos expansion for sensitivity analysis, Reliab. Eng. Syst. Saf. 94 (7) (2009) 1161-1172.

[44] A. Spinelli, M. Pini, V. Dossena, P. Gaetani, F. Casella, Design, simulation, and construction of a test rig for organic vapours, J. Eng. Gas Turb. Power 135 (2013) 042303.

[45] P.A. Thompson, Compressilbe Fluid Dynamics, McGraw-Hill, 1988.

[46] G. Gori, M. Zocca, G. Cammi, A. Spinelli, A. Guardone, Experimental assessment of the open-source SU2 CFD suite for ORC applications, Energy Procedia 129 (Supplement C) (2017) 256-263.

[47] C. Hirsch (Ed.), Numerical Computation of Internal \& Amp; External Flows: Fundamentals of Numerical Discretization, John Wiley \& Sons, Inc., New York, NY, USA, ISBN: 0-471-91762-1, 1988. 
[48] A. Dadone, B. Grossman, Surface boundary conditions for the numerical solution of the Euler equations, AIAA J. 32, http://dx.doi.org/10.2514/3. 11983.

[49] Z. Wang, Y. Sun, Curvature-Based Wall Boundary Condition for the Euler Equations on Unstructured Grids, Aiaa J. - AIAA J. 41, http://dx.doi.org/10. $2514 / 2.1931$.

[50] F. Cozzi, A. Spinelli, M. Carmine, R. Cheli, M. Zocca, A. Guardone, Evidence of complex flow structures in a converging-diverging nozzle caused by a recessed step at the nozzle throat, J. Chem. Eng. (2016).
[51] C.C. Conti, A. Spinelli, G. Cammi, M. Zocca, A. Guardone, Schlieren visualizations of non-ideal compressible-fluid flows., 13th International Conference on Heat Transfer, Fluid Mechanics and Thermodynamics.

[52] A. Spinelli, G. Cammi, M. Zocca, S. Gallarini, F. Cozzi, P. Gaetani, V. Dossena, A. Guardone, Experimental observation of non-ideal expanding flows of Siloxane MDM vapor for ORC applications, Energy Procedia 129 (2017) $1125-1132$. 